inconvénients potentiels de la méthode sont nombreux : la méthode est douloureuse et une anesthésie générale (ou au moins régionale) est nécessaire. Les ondes de choc sont très bruyantes. Elles peuvent causer des lésions tissulaires, notamment aux organes contenant de l'air (poumon et tube digestif) en raison de la différence d'impédance acoustique. Elles peuvent déterminer des troubles du rythme cardiaque si elles ne sont pas synchronisées avec l'électrocardiogramme. Enfin, le coût de la machine est élevé (elle a été qualifiée de baignoire la plus chère du monde).

Malgré ces inconvénients potentiels de la méthode et la prudence qui est encore, à l'évidence, nécessaire pour avoir une idée de ses possibilités et de ses indications, le très beau travail de l'équipe allemande ne va pas manquer de stimuler des études similaires ailleurs. Beaucoup de malades et de médecins en attendent déjà les résultats.

S. E.

I. Sauerbruch T, Delius M, Paumgartner G, et al. Fragmentation of gallstones by extracorporeal shock waves. $N$ Engl 7 Med 1986; 314: 818-22.

\section{Une fourmi}

\section{avec une seule}

\section{paire de chromosomes!}

Une fourmi australienne, Mirmecia pilosula, ne possède qu'une paire de chromosomes [I]. Les deux chromosomes des ouvrières sont homologues. Le mâle est haploïde et n'a qu'un chromosome. La seule autre espèce eucaryote connue jusqu'à présent pour n'être dotée que d'une paire de chromosomes est un nématode, Parascaris equorum univalens, et cela seulement dans la lignée germinale [2].

J.-C.D.

I. Crosland MWJ, Crozier RH. Mirmecia pilosula, an ant with only one pair of chromosomes. Science 1986; 231: 1278.

2. White WJD. Animal Cytology and Evolution.

\title{
Le GRF
}

Une mise au point sur le Facteur de libération de l'hormone de croissance (Growth hormone releasing factor, GRF) a été rócemment publiée par Fean Rossier [I] à la suite d'un Symposium qui s'est tenu à Toulouse, intitulé "GRF : Quo Vadis?". Au cours de ce Symposium, Roger Guillemin a rappelé l'histoire de la découverte du GRF. Il avait prédit qu'on trouverait ce peptide dans des tumeurs non hypophysaires accompagnées d'acromégalie et sécrétant des taux élevés d'hormone de croissance. C'est à la suite de l'observation à Lyon par Geneviève Sassolas d'une tumeur pancréatique répondant à ces critères que le peptide put être isolé et sa séquence déterminée dans le laboratoire de $R$. Guillemin (elle le fut en même temps dans celui de Vale à partir d'une autre tumeur du pancréas). On connaît aujourd'hui la séquence du GRF de six espèces de mammiferes. Elle compte le plus souvent 44 acides aminés, à l'exception de celle du rat qui a perdu l'acide aminé C-terminal. La partie indispensable à l'activité est le fragment GRF 3-2I. On pense qu'une substitution appropriée portant sur un seul acide aminé pourrait conduire à un antagoniste susceptible d'être utilisé priétés anti-insuliniques de l'hormone de croissance. Grâce au clonage, on a pu montrer que le produit primaire est un prépro GRF de 107 acides aminés, conduisant, à la suite de la coupure d'un peptide signal de 20 acides aminés, à un pro GRF de 87 acides aminés, précurseur du GRF définitif. $A$ l'aide d'anticorps spécifiques contre le GRF, Bertrand Bloch [2] a pu localiser le site de sécrétion du GRF, chez le singe écureuil et chez l'homme, dans les noyaux arqués et latéraux du tuber, dont les fibres se projettent sur l'hypothalamus. Les neurones à GRF sont distincts de ceux qui sécrètent d'autres peptides actifs de l'hypothalamus.

Un élément important de la sécrétion dans le diabète en raison des pro-

de lhormone de croissance est qu'elle est pulsatile, atteignant ses pics les plus élevés durant le sommeil et surtout les périodes de REM (mouvements oculaires rapides). Cette pulsatilité n'est pas supprimée par une perfusion continue de GRF. Elle semble résulter d'un balancement entre le $G R F$, qui stimule la sécrétion de l'hormone de croissance et la somatostatine, qui l'inhibe [3].

Un problème capital pour l'avenir est celui de l'importance pathologique et éventuellement thérapeutique $d u$ GRF : le nanisme hypophysaire est-il dî à un défaut primitif en hormone de croissance ou en GRF? Un grand nombre de "nains hypophysaires" pourraient être en fait des "nains hypothalamiques". Un élément décisif réside dans la réponse de Phormone de croissance au GRF de synthèse. Celle-ci semble très bonne, $\grave{a}$ condition de ne pas se laisser décourager par l'absence d'effet d'une dose unique de GRF; l'existence d'une période de latence est la règle dans les déficits anciens en hormone de croissance, et la réponse s'améliore progressivement lorsqu'on poursuit les injections de GRF. De grands espoirs peuvent donc être nourris pour l'avenir.

Dans ses conclusions, 7. Rossier estime enfin que la quête des facteurs hypophysiotropes, déclenchée il y a 20 ans par l'isolement du facteur de libération de l'hormone thyréotrope (TRH), puis de l'hormone de libération des gonadotrophines ( $\mathrm{GnRH}$ ) et de la somatostatine, devrait être désormais considérée comme close.

J.-C. D.

1. Rossier J. GRF update: is this the last hypothalamic hypophysiotropic factor? Trends Neurosci 1986; 9: 95-6.

2. Bloch $B$, Barzeau $P$, Ling $N$, et al. Immunohistochemical detection of growth hormone releasing factor in brain. Nature 1983; 301: 607-8. 3. Vance ML, Kaiser DL, Evans WS, et al. Pulsatile growth hormone secretion in normal man during a continuous 24-hour infusion of human growth hormone realeasing factor. $7 \mathrm{Clin}$ Invest 1985; 75: $1584-90$.

Note de dernière minute. Un défaut de fonctionnement du récepteur au GRF pourrait être une cause de nanisme; cela semble le cas des souris naines de la souche "little , dont la sécrétion d'hormone de croissance ne répond pas au $G R F$, mais répond ì $T A M P$ cyclique.

Fansson 7O, Downs TR, Beamer $W$, Frohman $L A$ Receptor-associated resistance to grodth-hormone releasing factor in dwarf "little" mice. Science 1986, $232: 511-2$. 